\title{
ARTÍCULO
}

\section{Derecho a la salud de mujeres en contexto de prostitución en Sevilla} (Andalucía). Más allá de la salud sexual.

\section{Health as a right in women who are in context of prostitution in Sevilla (Andalucía). Beyond sexual health.}

\author{
Rafael Barroso-Pavía \\ Doctorando Ciencias Jurídicas y Políticas \\ Universidad Pablo de Olavide \\ Nuria Cordero Ramos \\ Departamento de Trabajo Social y Servicios Sociales \\ Universidad Pablo de Olavide
}

Fecha de recepción 02/08/2017 | De aceptación: 06/06/2018 | De publicación: 27/06/2018

\section{RESUMEN.}

El presente trabajo hace una aproximación al derecho a la salud en las mujeres que ejercen la prostitución en Sevilla (Andalucía). Para ello contextualizaremos la situación económico-social en la cual se encuentra nuestro país en este momento y sus influencias en el Derecho a la salud. A continuación, analizaremos la relevancia de la salud como instrumento de inclusión/exclusión social, para después hablar de la situación de salud y vulnerabilidad de las mujeres en contexto de prostitución en Sevilla. A modo de cierre se presentan algunas reflexiones sobre el camino a seguir para avanzar en el derecho a la salud de este colectivo.

\section{PALABRAS CLAVE.}

Derecho a la salud; Prostitución; Mujeres; Prostitutas; Inclusión social; Exclusión social

\section{ABSTRACT.}

This paper makes an approximation to the right to health in women who are practising prostitution in Sevilla (Andalucía). Firstly, we are going to contextualize the social and economic situation in Spain and how it has affected in the health right. Next, we are going to analyse the importance of this right as an instrument for social inclusion/exclusion and we will discuss the health situation and vulnerability of women in prostitution. Finally, we will present our opinion about the correct protection of this right for this group.

\section{KEY WORDS.}

Health as a right; Prostitution; Women; Prostitutes; Social inclusion; Social exclusion 
Sumario: 1. Introducción. 2. Derecho a la salud: El caso de Andalucía. 3. La salud como instrumento de inclusión/exclusión social. 4. Salud y vulnerabilidad de las mujeres que están en contexto de prostitución en Sevilla. 5. A modo de cierre.

\section{Introducción.}

La finalidad de este trabajo es la de aproximarnos a las dificultades para acceder a la salud de aquellas mujeres que se encuentran ejerciendo la prostitución. Analizaremos el estado de dicho derecho en nuestro país, profundizando en la realidad de Andalucía y, concretamente, la de Sevilla. Todo ello con la intención de poder observar cuál es el marco en el que nos ubicamos. Tras dicho análisis, argumentaremos la relevancia que posee el derecho y el acceso a la salud como un mecanismo de inclusión/exclusión - a través de diferentes determinantes -. Finalizaremos con el estado de la salud y la vulnerabilidad de las mujeres que están en contexto de prostitución en Sevilla, exponiendo algunas percepciones, de las propias mujeres y técnicos y voluntarios de distintas ONGs que atienden a este colectivo, sobre cuestiones relacionadas con la salud.

Para ello, se ha realizado una revisión bibliográfica sobre estudios ya realizados sobre el acceso a la salud en general y, también, sobre la salud de este colectivo en concreto. También se ha analizado el desarrollo legislativo y de políticas públicas que este derecho posee en el contexto donde estas mujeres ejercen la prostitución, para así poder comprobar si lo recogido en las políticas y normas desarrolladas, coincide con la realidad a la que ellas tienen que hacer frente en su día a día. Además, desde el año 2015 venimos realizando observación del grupo de estudio, a través de la colaboración en diferentes programas de voluntariado de diversas ONGs que trabajan con el colectivo. Ello nos ha permitido aproximarnos a la realidad de algunas de estas mujeres y conocer algunas percepciones y opiniones sobre esta cuestión.

Al ser el derecho a la salud el tema central de este trabajo, nuestra primera labor debe ser determinar qué entendemos por salud. En este sentido, tomamos como punto de partida la definición de salud que propone la Organización Mundial de la Salud, la cual la define como "un estado de completo bienestar físico, mental y social, y no solamente la ausencia de afecciones o enfermedades" (Organización Mundial de la Salud, 2014, pp. 1).

Tomando como referencia esta definición nos disponemos a explicar cómo se promueve el derecho a la salud. En este sentido, hacemos referencia al Estado de Bienestar como uno de los principales fundamentos para el desarrollo de los diferentes marcos legales en Europa después de la Segunda Guerra Mundial (Burgaya, J. 2013), y 


\section{CEFD}

Cuadernos Electrónicos de Filosofía del Derecho

que es considerada una de las principales victorias en las sociedades occidentales europeas. A través de esta idea, se desarrolló el concepto de Estado Social de Derecho que ha llegado hasta nuestros días. La intervención del Estado en las cuestiones de ámbito social como una obligación germinó en el reconocimiento de derechos sociales que, posteriormente, se recogieron en las diferentes Constituciones (Marcos del Cano, A. M. \& Méndez, Martín, J. 2016).

Sin embargo, con la llegada de la crisis económica a finales de la primera década del año 2000, resurgió el conflicto respecto a la viabilidad del Estado de Bienestar, así como a la amplitud que su actuación debía tener (Mar del Cano, A. M. \& Méndez, Martín, J. 2016). Ello se ha traducido en una serie de recortes y limitaciones a estos derechos, implicando un descontento casi universal en la sociedad que ha visto como, en general, ha mermado su acceso a ciertos servicios.

Pero cuando hablamos de derecho a la salud, no sólo hacemos referencia a un derecho social, sino también a un derecho humano, puesto que así viene recogido en la Declaración Universal. El art. 25 de la DUDDHH ${ }^{1}$ hace alusión, entre otras cuestiones, a que toda persona tiene derecho a que se le asegure la salud y el bienestar, así como a la

\footnotetext{
${ }^{1}$ Declaración Universal de Derechos Humanos.
}

asistencia médica y los servicios sociales necesarios.

Además de cuestiones legales, el alcance o limitación en el acceso al derecho a la salud está atravesado por cuestiones relacionadas con el género, ya que existen obstáculos de accesibilidad para las mujeres en relación con los hombres (Panisello, M. L. \& Pastor Gosalbez, M. I., 2015). Siendo esta una realidad que debe tenerse en cuenta a la hora de abordar las diferentes enfermedades, barreras y escenarios donde se ha de intervenir desde las políticas públicas. Todo ello con el fin de reducir las desigualdades sociales y combatir la exclusión que padecen diversos colectivos de mujeres, como son las que están ejerciendo la prostitución.

A lo largo de las siguientes páginas hacemos una aproximación a dicha realidad, teniendo presentes los marcos reguladores del derecho a la salud en Andalucía, así como la heterogeneidad que existe dentro del propio colectivo, aunque todas comparten limitaciones ${ }^{2}$ para acceder a este derecho por el hecho de ejercer la prostitución.

\section{Derecho a la salud: El caso de Andalucía.}

\footnotetext{
${ }^{2}$ En ocasiones, estas limitaciones son producto del temor a que se conozca la actividad a la que se dedican y las consecuencias que ello pueda tener en su vida cotidiana, mientras que en otros casos, como el de aquellas mujeres extranjeras, las limitaciones son consecuencia de la situación legal que poseen en nuestro país y cómo les impide acceder a determinados servicios.
} 


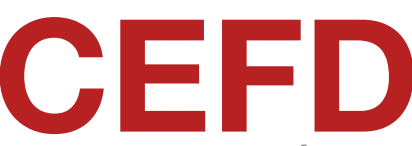

Cuadernos Electrónicos de Filosofía del Derecho

El derecho a la salud fue una de las grandes conquistas en la lucha de derechos sociales en el denominado Estado del Bienestar. El reconocimiento del derecho a la protección de la salud puede encontrarse en numerosas normas, tal y como hemos mencionado anteriormente. En el ámbito internacional, nos encontramos con su presencia en el artículo 25.1 de la DUDDHH.

En el caso de España, el Derecho a la Salud o, mejor dicho, el derecho a la protección de la salud se encuentra recogido en el artículo 43 de la Constitución. Pero para poder comprender mejor en su totalidad la amplitud de este derecho, debemos fijarnos, también, en el artículo 53.3 de la misma.

De la lectura de estos artículos podemos sacar las siguientes conclusiones:

En primer lugar, que estamos ante un Derecho Humano, reconocido de forma internacional por todos aquellos países signatarios de la Declaración, los cuales, además, se comprometen a respetarla.

En segundo lugar, en el caso de los artículos desarrollados en la Constitución, observamos que la salud, o su acceso, es un derecho constitucionalizado, cuya protección quedará determinada por la regulación que se realice de este derecho por parte de los poderes públicos, siendo, por tanto, un derecho condicionado a la voluntad del legislador. Además, se estipula que será labor de los distintos poderes públicos el fomentar la educación sanitaria, por lo que se entiende que las instituciones trabajarán esta cuestión de educación a través de la elaboración de sus políticas públicas. Por tanto, hablamos de un derecho condicionado por la legislación que se haga de él, poseyendo una protección más limitada.

Pero este derecho fundamental posee otra característica relevante, que se recoge en los artículos 148 y 149 de la Constitución. En estos artículos se establece que las Comunidades Autónomas tendrán capacidad, entre otras materias, en Sanidad. Esto hace que el desarrollo y gestión de este derecho se traslade a las diferentes autonomías que conforman nuestro país, provocando una diversidad de marcos reguladores sobre este derecho. $\mathrm{Si}$ bien el Gobierno central puede desarrollar cuestiones respecto a él, son las Comunidades Autónomas las que acaban aplicando las directrices que el Gobierno central desarrolla en la medida que estiman oportuno.

En el caso de Andalucía, dentro de la gestión realizada por el Gobierno andaluz, se han aprobado las siguientes normas: la Ley de Salud Pública de Andalucía (2011); el IV Plan Andaluz de Salud (2013); el Anteproyecto de Ley de Garantías y Sostenibilidad del Sistema Sanitario Público de Andalucía (2015); y, finalmente, la 
Finalmente, la Carta de Derechos y Deberes en de Filosofía del Derecho

Carta de Derechos y Deberes en Salud (1998), entre otras. Elegimos estas ya que creemos que analizan las cuestiones básicas relacionadas con la protección de la salud en Andalucía.

En la Ley de Salud Pública de Andalucía se establece la salud y la garantía para su protección como una materia transversal y sitúa a la ciudadanía como el eje central, fomentando su uso responsable y distribuyendo las competencias en este sector - entre otras cuestiones -.

El IV Plan Andaluz de Salud, dedica sus páginas a cuestiones como cuál es su punto de partida, la situación de la salud en Andalucía (relacionándolas con los aspectos demográficos, los condicionantes sociales de la salud, los factores de riesgo, la situación de la salud y las actividades y perspectivas del sistema sanitario), hacia donde quiere avanzar, así como los compromisos que realiza, finalizando con las pautas para su puesta en marcha, la necesidad de implicación de todos los sectores y cómo deberá evaluarse.

En relación con el Anteproyecto de Ley de Garantías y Sostenibilidad del Sistema Sanitario Público de Andalucía, la Junta pretende garantizar $y$ reforzar los derechos constitucionales $y$ estatutarios en materia de protección de la salud para cualquier persona, basándose en los principios de universalidad, equidad, solidaridad social e igualdad. 
- En relación con la aplicación de las reformas legales, la Junta las ha desarrollado de forma limitada.

- En Andalucía no llegó a aplicarse la limitación de acceso a la población sin permiso de residencia desarrolladas por el RD 16/2012. A pesar de ello, este dato es desconocido para muchas de las personas que se ven afectadas por esta condición, no accediendo a estos servicios mujeres migrantes que lo necesiten. No obstante, desde diferentes ONGs que trabajan con este colectivo se les informa de ello ${ }^{3}$.

- Por otro lado, la privatización sanitaria en Andalucía ha seguido un aumento medio respecto al de otras Comunidades, mientras que la financiación y los recursos sanitarios ha disminuido intensamente ${ }^{4}$.

La repercusión de la crisis económica en la salud ha provocado una disminución, de forma considerable desde el año 2009, del gasto en salud, como destaca Segura Benedicto, A. (2014) agravando de forma directa e indirecta cuestiones relacionadas con el acceso a la salud y su protección. De forma directa son de destacar los numerosos recortes presupuestarios y las medidas

\footnotetext{
${ }^{3}$ Información obtenida de los técnicos de ONGs como Médicos del Mundo y Cruz Roja en Sevilla.

${ }^{4}$ Para profundizar en esta idea consultar la obra de Bacigalupe, A. et al. (2016).
}

legales y de austeridad destinadas al sector sanitario. De forma indirecta, estas medidas han afectado a las condiciones de $\operatorname{vida}^{5}$ de la ciudadanía, teniendo una mayor repercusión en aquellas cuestiones que afectan al nivel adquisitivo así como a la situación laboral y las condiciones de trabajo.

Más relacionado con el tema central de este trabajo, el derecho a la salud de las mujeres que se encuentran en contexto de prostitución, a nivel autonómico encontramos un convenio de salud entre la Junta y este colectivo. En dicho convenio, se garantiza la atención a la salud de las personas que ejercen la prostitución. En la guía desarrollada por la Junta de Andalucía ${ }^{6}$, datada en 2007, encontramos que la vía que la Junta elige para garantizar este derecho se basa en la colaboración de los servicios sanitarios con diferentes ONGs. Sin embargo, en el año 2015 se implanta un nuevo protocolo de actuación dedicado a la violencia de género $^{7}$ y en el que encuadran la prostitución dentro de un capítulo dedicado a la "actuación sanitaria con mujeres en

\footnotetext{
${ }^{5}$ Definido por el art. 2 de la Ley de Salud Púbica de Andalucía como aquel entorno cotidiano de las personas, donde estas viven, actúan y trabajan, y que son productos de las circunstancias sociales y económicas, así como del entorno físico, todo lo cual puede ejercer impacto en la salud, estando en gran medida fuera del control inmediato del individuo.

http://www.juntadeandalucia.es/salud/servicios/contenidos/andaluc iaessalud/docs/31/AtencionProstitucion FINAL.pdf 7

http://www.juntadeandalucia.es/salud/sites/csalud/contenidos/Infor macion General $/ \mathrm{p} 2$ p 2 planes integrales/protocolo violencia genero?perfil $=$ org
} 
relación directa con las lógicas que sostienen la

Cuadernos Electrónicos de Fillosofía del Derecho

situaciones especiales" y al que le dedican una página de extensión, centrándose sobre todo en la mutilación genital y la trata. Al igual que el convenio anterior, centra su actuación en el trabajo conjunto de los servicios de salud y las ONGs, fomentando la educación sexual y la prevención de ITS, así como otros temas de índole socioeconómico para fortalecer las redes sociales y darles alternativas de empleo. No obstante, la práctica real difiere de la establecida, encontrándonos en numerosas ocasiones con el desconocimiento por parte de las instituciones y de las propias mujeres sobre estos derechos.

Además, este sector también se ha visto afectado por la crisis económica, la cual ha provocado un aumento en el número de mujeres españolas que ejercen prostitución, así como de extranjeras que la habían dejado hace años, y que no han encontrado otra salida al quedarse sin trabajo y sin prestaciones (Cáritas Española, 2016),

En el siguiente apartado profundizaremos sobre la influencia de la salud como un instrumento de inclusión/exclusión social con este colectivo.

\section{La salud como instrumento de}

\section{inclusión/exclusión social.}

Como se viene enunciando, las posibilidades de acceder a la salud que tienen las mujeres que están en contexto de prostitución, están en dicotómica relación de inclusión/exclusión. Faura Martínez, U. \& Lafuente Lechuga, M. (2012) apuntan que el concepto de exclusión social no hace referencia sólo a desigualdades latentes en el ámbito económico, sino que amplía su ámbito a diferentes áreas que completan nuestro día a día, calificadas como "resultado de una cadena de acontecimientos derivados de las desigualdades económicas y sociales" (Pp. 177).

El Plan Nacional para la Inclusión Social del Reino de España 2013-2016 (2014) determina que los principales factores que producen una mayor influencia en la pobreza y la exclusión social ${ }^{8}$ son el empleo/desempleo, la educación, la vivienda y, finalmente, la salud. En cuanto a la salud, el Plan destaca la existencia de un pequeño porcentaje de la población que declara haber encontrado algún tipo de barrera a la hora de acceder a estos servicios, debido a cuestiones temporales, económicas, etc. Además, el Plan determina que este tipo de situaciones se encuentra con mayor presencia en determinados grupos de población, siendo uno de ellos el formado por personas que se encuentran excluidas del mercado laboral - en el cual podríamos incluir, por el reconocimiento que la prostitución posee en nuestro país, a

\footnotetext{
${ }^{8}$ Definiendo exclusión social como un "proceso de pérdida de integración o participación de las personas en la sociedad y en los diferentes ámbitos económico, político y social" (Pp. 10).
} 


\section{CEFD}

Cuadernos Electrónicos de Filosofía del Derecho

aquellas mujeres que se dedican a esta actividad -

Algunas de estas mujeres, que ven mermada su posibilidad de acceso al mercado de trabajo, utilizan esta actividad como un medio de subsistencia para ellas y para sus familias. Según el Centro de Inteligencia contra el Crimen Organizado, en España hay aproximadamente 45.000 mujeres ejerciendo la prostitución (Ministerio de Sanidad, Servicios Sociales e Igualdad, 2015, pp. 37). Sin embargo, la relación de la prostitución con ciertas conductas delictivas, unida a las características de clandestinidad y consiguiente invisibilidad, así como la estigmatización y la exclusión social en que se encuentran las personas en situación de prostitución, dificulta en gran medida la obtención de datos, sin que existan hasta el momento estadísticas oficiales al respecto.

En el caso de mujeres en contexto de prostitución, autoras como MacKinnon, C. A. (2010) alegan que el género es una cuestión fundamental a la hora de comprender dicha actividad. Esta idea es, en parte, secundada por otras autoras como Pateman, C. (1990), para la cual la conexión entre el sexo y el poder sólo puede entenderse por medio del estudio del género.

Al igual que otros ámbitos como el trabajo, el estado civil, la clase social o el nivel educativo, el género es un determinante de la salud ${ }^{9}$ para tener en cuenta a la hora de aprobar políticas públicas, desarrollar diagnósticos o proponer un tratamiento.

La cuestión del género se encuentra dentro de los denominados ejes de desigualdad, concretamente en los determinantes estructurales de las desigualdades en salud. Panisello, M. L. \& Pastor Gosalbez, M. I. (2015), incluyen este concepto en la protección para poder diseñar políticas sanitarias que puedan mitigar las desigualdades que existen, ya que posee una gran conexión "en el proceso salud-enfermedad, produciendo desigualdades (en salud) ${ }^{10}$ que se manifiestan en el acceso a los hospitales, las listas de espera y las estrategias terapéuticas" (Pp. 1556).

Como podemos comprobar, la presencia o no de desigualdades en salud ${ }^{11}$ se encuentra fuertemente relacionada con la existencia de inclusión o exclusión. Los diferentes determinantes que la componen se resumen a la perfección en la siguiente figura:

\footnotetext{
${ }^{9}$ Definido como el conjunto de factores que determinan el estado de salud individual y colectiva (Art. 2 Ley de Salud Pública de Andalucía).

10 Entendida como las diferencias que perjudican, de manera sistemática e injusta, a una persona o grupo en término de oportunidades y que originan una merma en su situación de salud (Art. 2 Ley de Salud Pública de Andalucía).

11 Definida por la Comisión para Reducir las Desigualdades Sociales en Salud en España (2012) como "aquellas diferencias en salud injustas y evitables entre grupos poblacionales definidos por sus aspectos sociales, económicos, demográficos o geográficos. (...) son el resultado de la distinta distribución de circunstancias, oportunidades y recursos relacionados con la salud que tienen las personas, y que se traduce en una peor salud entre los colectivos socialmente menos favorecidos" (Pp. 183).
} 


\section{CEFD}

Cuadernos Electrónicos de Filosofía del Derecho

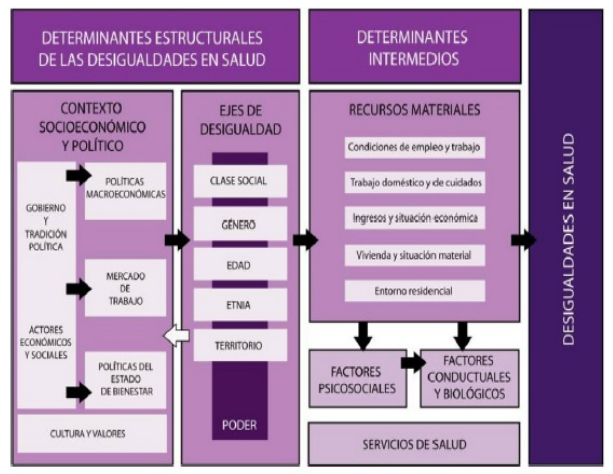

Figura 3. Marco conceptual de
en Solare elrwin ${ }^{3}$ Navarro $0^{16}$

Fuente: Comisión para Reducir las

Desigualdades Sociales en Salud en España (2012), Pp. 184.

Del análisis de dicha figura obtenemos las siguientes percepciones:

- Los determinantes que pueden influir en la salud pueden ser estructurales y/o intermedios.

- Dentro de los determinantes estructurales diferenciamos el contexto socioeconómico y político, conformado por las actuaciones llevadas a cabo en la esfera macro, como, por ejemplo, las políticas desarrolladas por el Estado o la situación del mercado laboral; así como los denominados ejes de desigualdad, considerados más personales y que pueden influir en la mayor o menor posibilidad de sufrir desigualdades.

- Por otro lado, los determinantes intermedios estarían compuestos por los recursos materiales a los que las personas pueden acceder y que se complementan con los ejes de desigualdad. Dentro de este grupo destacan los factores psicosociales, así como los conductuales y biológicos.

Todas estas cuestiones, unidas al acceso a los servicios de salud y la percepción que se tenga de dichos servicios, van a condicionar las desigualdades en salud de la ciudadanía.

En el caso de Andalucía, encontramos que, tal y como recoge Silva Ardanuy, F. M. (2015) en su obra, el gobierno de la Comunidad lleva a cabo un reconocimiento de estas cuestiones de inclusión/exclusión en el desarrollo de sus políticas y recoge, mediante el desarrollo de normativas específicas, la lucha contra la exclusión social. Aun así, cabe cuestionarnos hasta qué punto este avance puede ser encontrado en la práctica que, finalmente, se lleva a cabo.

La importancia del acceso a este derecho pasa por potenciar los mecanismos que favorezcan la inclusión y combatan la exclusión, protegiendo así la finalidad de lograr una sociedad más inclusiva. Aún queda mucho trabajo por hacer, pues es necesario el profundizar y desarrollar aspectos más concretos para colectivos específicos, como el central de nuestro trabajo, las mujeres en contexto de prostitución.

4. Salud y vulnerabilidad de las mujeres que están en contexto de prostitución en Sevilla. 


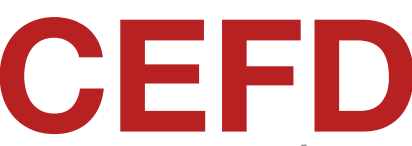

Cuadernos Electrónicos de Filosofía del Derecho

Una vez esbozado el contexto en el que se encuentra la salud, es momento de aproximarnos a la realidad de las mujeres que ejercen la prostitución en la ciudad de Sevilla. La metodología utilizada ha sido cualitativa, utilizando como técnicas principales la observación participante en calle, clubs y en las distintas sedes de trabajo de organizaciones que se dedican a la atención de este colectivo, así como entrevistas semiestructuradas, realizadas tanto a profesionales como a las mujeres. La finalidad ha sido la de acceder a sus opiniones y percepciones sobre el derecho a la salud, así como observar las condiciones en las que desarrollan la actividad y los riesgos a los que se enfrentan. El acceso a las mujeres y a los lugares donde ejercen ha sido posible gracias a la colaboración voluntaria que venimos desempeñando en varias organizaciones desde 2015 hasta el momento actual. A través de este trabajo hemos tenido acceso, aproximadamente, a 50 mujeres, de forma continua, número que podría elevarse si contamos con las atendidas de forma discontinua (ya que no las hemos vuelto a ver más en los lugares habituales que visitan las diversas organizaciones). Al mismo tiempo, hemos tenido acceso a profesionales, especialmente los responsables de dos entidades que tienen programas que trabajan con este colectivo en Sevilla, y voluntarios de estas que se dedican a la atención directa con las mujeres tanto en la calle y clubs como en las sedes institucionales.

Conviene resaltar que la actividad principal de las entidades que atienden a este colectivo se basa en el traslado del personal técnico y voluntariado a clubs y a lugares habituales de ejercicio de prostitución para ofrecer apoyo a las chicas y hacer entrega de un pack con material para el correcto desarrollo de su actividad (compuesto, principalmente, por preservativos - tanto masculinos como femeninos -, lubricante y toallitas de higiene íntima), así como ofrecer información, que dichas entidades consideran que puede ser de su interés, (esencialmente cuestiones relacionadas con la salud sexual, aunque también otras vinculadas con la obtención de prestaciones y ayudas por parte de los entes públicos).

Respecto a las mujeres, como ya se ha mencionado, no existe un perfil único, sino que observamos una gran variedad, que, además dependiendo, de su procedencia de país de origen, se encuentran principalmente en calle o clubs. La mayoría de las mujeres a las que hemos accedido son extranjeras, aunque también se detectaron mujeres españolas, localizándose, en su mayoría, dentro del grupo de mujeres que ejerce la prostitución en la calle. En el caso de las mujeres extranjeras, predominan tanto en la prostitución de clubs como en la de calle, siendo la mayoría de Europa del Este, Latinoamérica y África; mientras que las españolas son de la ciudad o alrededores, 


\section{CEFD}

Cuadernos Electrónicos de Filosofía del Derecho

aunque también hay casos de mujeres procedentes de otros puntos de España, pero que llevan años asentadas en la zona.

Las percepciones y opiniones sobre salud, tanto de las mujeres como de los profesionales $\mathrm{y}$ voluntarios a los que hemos tenido acceso en el trabajo de campo, se pueden sintetizar de la siguiente forma:

$\underline{\text { Salud sexual }}{ }^{12}$. Encontramos una predominancia sobre este tipo de cuestiones. Las propias mujeres consideran que poseen un buen nivel de salud, y se muestran preocupadas y concienciadas en cuanto a su salud sexual. Algunas de ellas llevan un control estricto, mientras otras se muestran más esquivas a hablar del tema $\mathrm{y}$ aseguran llevar un control, aunque más laxo consideramos que como consecuencia de temores personales y desconocimientos -. Estimamos que existe una influencia positiva hacia el cuidado personal, en parte gracias a la labor continua que desempeñan las organizaciones que ofrecen, además, programas de educación sobre salud sexual.

\footnotetext{
12 Definida por la OMS como "un estado de bienestar fisico, mental y social en relación con la sexualidad. Requiere un enfoque positivo y respetuoso de la sexualidad y de las relaciones sexuales, así como la posibilidad de tener experiencias sexuales placenteras y seguras, libres de toda coacción, discriminación y violencia". (http://www.who.int/topics/sexual health/es/)
}

$\underline{\text { Salud mental }}{ }^{13}$. Muchas de las mujeres presentan síntomas de ansiedad, estrés, incluso depresión, aunque estas cuestiones de salud no suelen trabajarse. Además, este tipo de cuestiones no son abordadas de forma directa, sino que en la mayoría de los casos, según los profesionales, se observa por la sintomatología que presentan y, en otros, se dejan entrever en las conversaciones que se mantienen con ellas. Es en casos de extrema presión cuando demandan la cobertura de este tipo de necesidades, relacionadas con cuadros de ansiedad, casos de abusos y violencias, problemas emocionales, etc. Si bien es cierto que se les presenta la opción de recibir ayuda psicológica, no suele ser la ayuda más demandada, aunque sí que la mencionan como algo necesario en momentos puntuales.

Adicciones $^{14}$. Otra de las cuestiones que se plantean en relación con la salud, se centra en las adicciones en relación con el consumo de drogas, alcohol y fármacos. Estas adicciones se han evidenciado en algunas de las mujeres que ejercen en la calle, siendo la principal adicción

\footnotetext{
${ }^{13}$ Definida por la OMS como "un estado de bienestar en el cual el individuo es consciente de sus propias capacidades puede afrontar las tensiones normales de la vida, puede trabajar de forma productiva y fructífera y es capaz de hacer una contribución a su comunidad".

(http://www.who.int/features/factfiles/mental_health/es/)

14 Definida por la OMS como "el consumo repetido de (...) sustancias psicoactivas, hasta el punto de que el consumidor se intoxica (...) de forma continua, muestra un deseo compulsivo de consumir (...), tiene una enorme dificultad para interrumpir voluntariamente (...) el consumo de la sustancia y se muestra decidido a obtener(las) por cualquier medio". (http://www.who.int/substance abuse/terminology/lexicon alcoho 1 drugs spanish.pdf)
} 


\section{CEFD}

Cuadernos Electrónicos de Fillosofía del Derecho

manifestada el consumo de determinados estupefacientes. Por lo general, aquellas mujeres con adicciones son conscientes de su problema y manifiestan su deseo de abandonar el consumo, aunque, al mismo tiempo, reconocen que las circunstancias y contextos en los que se encuentran viviendo no les ofrece oportunidades favorables para ello.

Condiciones en las que ejercen la actividad. Encontramos una diferencia clara entre las condiciones de las mujeres que ejercen en calle y las que ejercen en clubs, detectando también grandes diferencias entre las condiciones de los clubs en cuanto a luminosidad, higiene, accesibilidad, etc. En general, las mujeres que ejercen en calle han expresado dificultades para desarrollar su actividad con seguridad, ya que se veían obligadas a trasladarse a polígonos industriales alejados y con poco tránsito. En cuanto a las mujeres que ejercen en clubs, encontramos que las condiciones variaban respecto al club, aunque por lo general manifestaban cansancio por tener que adecuarse a los horarios así como incomodidad por tener que utilizar la misma habitación donde dormían y descansaban como lugar donde realizar el servicio, sin favorecer la desconexión de su vida personal de la actividad que realizan.

Además, se constata la violencia como un factor transversal a los aspectos destacados anteriormente $\mathrm{y}$ que se manifiesta con gran relevancia a la hora de entender su estado de salud. Y es que está fuertemente conectado con situaciones que pueden llegar a sufrir y que condicionan el buen desarrollo de la misma. Estas violencias no pueden ser entendidas sólo como aquellas que pueden llegar a sufrir por los clientes de sus servicios, sino que debemos mantener una visión más amplia y encuadrar también el abuso de poder que en ocasiones sufren por parte de las autoridades, las restricciones que pueden llegar a tener para acceder a los servicios sanitarios y la presión que conlleva el ejercicio de la actividad y su conciliación con la vida familiar.

Por otro lado, los recortes que se han producido en determinadas políticas públicas han ocasionado una disminución en la atención que determinadas organizaciones realizan con este colectivo, así como el acceso a otros servicios que complementan la labor que estas organizaciones llevan a cabo.

A todos estos obstáculos, hay que sumarles:

- Su condición de inmigrante. Las situaciones de irregularidad en la que se encuentran muchas mujeres hacen que tengan miedo de utilizar los recursos públicos.

- Su desconocimiento de la normativa de nuestro país, que limita su acceso a los servicios sanitarios, por dos causas: la 
primera, por desconocer la limitación del RD 16/2012 en la Comunidad de Andalucía; y la segunda, por no saber cómo poder solicitar su tarjeta sanitaria (qué documentación es necesario aportar, dónde deben solicitarlo... $)^{15}$.

- La percepción social de la prostitución, lo cual hace que estas mujeres sean criminalizadas y no se sientan cómodas al acceder a los servicios públicos (ya sean de índole sanitario, social, de seguridad, etc.) y comentar la actividad que desarrollan (viéndose esa incomodidad aumentada en el caso en que son madres, por miedo a que ello llegue a conocimiento de los Servicios Sociales y puedan ser separadas de sus hijos).

Esta aproximación a la realidad deja entrever algunos de los principales obstáculos a los que se enfrentan a la hora de intentar mantener un buen estado de salud, el cual se ve condicionado por el hecho de ser mujeres, en algunos casos extranjeras, que ejercen la prostitución totalmente expuestas y sin apenas protección.

\section{A modo de cierre.}

\footnotetext{
${ }^{15}$ Si bien, con la llegada al gobierno del PSOE tras la moción de censura, se ha manifestado la intención de volver a implantar la sanidad universal, lo cual conllevaría la eliminación de esta restricción ya no existiera.
}

Para finalizar, esbozaremos algunas líneas de trabajo necesarias para continuar avanzando en la tarea de garantizar el derecho a la salud a las mujeres en contexto de prostitución.

Tal y como se ha venido exponiendo, la crisis económica ha provocado recortes en las políticas públicas de protección y el aumento de mujeres que ejercen la prostitución como medida de subsistencia. Los distintos gobiernos, en sus diferentes niveles, han tomado decisiones económicas y políticas que se han traducido en una reducción considerable del presupuesto destinado a este sector, así como en los servicios prestados. Esto ha provocado que el derecho a la salud se vea comprometido. Además, en el caso de las mujeres en contexto de prostitución, estos recortes les afectan desde el acceso a los servicios sanitarios por las grandes esperas que pueden producirse, a las atenciones que les son prestadas por entidades y organizaciones que se han visto afectadas por importantes reducciones en sus partidas.

La accesibilidad a los servicios sanitarios se ha visto comprometida, por cuestiones administrativas - como la necesidad de poseer tarjeta sanitaria para acceder a determinados recursos, la obligación de estar empadronadas para poder solicitar dicha tarjeta... - y por restricciones en las políticas migratorias, afectando de forma directa a numerosas mujeres 


\section{CEFD}

Cuadernos Electrónicos

de Filosofía del Derecho

migrantes que forman parte del colectivo $^{16}$. Además, el desconocimiento de la normativa por parte de algunas de estas mujeres se ha traducido en una mayor desprotección que tiene consecuencias tanto en su salud física como mental ${ }^{17}$.

Resulta necesaria una concienciación, más amplia, sobre los riesgos a los que se enfrentan en el ejercicio de la actividad, reconociendo la importancia de la salud mental para minimizar las consecuencias del estigma, la criminalización ${ }^{18}$, así como de las violencias y la exclusión, que afecta a buen sector de estas mujeres. Por ello, junto a la concienciación es necesario garantizar una atención integral y un seguimiento, dándoles protagonismo y escuchando sus propuestas con el fin de que puedan acceder más fácilmente a los recursos existentes.

Como se ha venido exponiendo, las políticas públicas destinadas a minimizar la exclusión y a garantizar el derecho a la salud han de continuar profundizando en el análisis de los determinantes de salud y/o enfermedad en relación con el género

\footnotetext{
16 Datos que corroboran lo dicho por Cáritas (2016) e Instituto Andaluz de la Mujer (2005).

${ }^{17}$ Frecuentemente, ellas manifiestan un gran nivel de agotamiento y de cansancio, asociado a la actividad que realizan y a las condiciones que tienen para desarrollarlas. Además, el ocultarlo a sus familiares y seres queridos más cercanos, así como el encontrarse lejos de ellos, en el caso de las mujeres extranjeras, hace que la presión sea mayor y que su salud psicosocial se encuentre condicionada
}

${ }^{18}$ Tal y como destaca la autora Maqueda Abreu, M. L. (2017). y la actividad de la prostitución con el fin de prevenir los factores de riesgo y actuar favoreciendo la accesibilidad a los recursos públicos de salud disponibles. Al mismo tiempo, resulta necesario fomentar programas formativos para el personal sanitario, operadores jurídicos y cuerpos y fuerzas de seguridad donde se aborden cuestiones relacionadas con la sensibilidad de género, diversidad cultural y proximidad a la gran variedad de realidades del colectivo, minimizando las visiones estereotipadas de las mujeres en contexto de prostitución y favoreciendo el acceso a la salud ${ }^{19}$.

Desde el trabajo académico, tanto en la línea jurídica como sanitaria, son necesarias investigaciones aplicadas sobre la temática que incluyan la transversalidad ${ }^{20}$, para conseguir avanzar no sólo en las explicaciones y las categorizaciones sino también en garantizar coberturas frente a los obstáculos a los que se enfrentan estas mujeres en su cotidianidad.

Con el fin de minimizar los riesgos de vulnerabilidad de estas mujeres se debe facilitar información y formación a la ciudadanía sobre el trabajo sexual y sus consecuencias para las

\footnotetext{
19 Esta demanda llega tanto del contacto directo con ellas, así como de la recopilación de sus demandas por parte de otras autoras como López Precioso, M. \& Mestre i Mestre, R. M. (2007).

${ }^{20}$ Entendiendo la transversalidad como Esteban, M. L. (2006), en referencia a un diálogo entre las diferentes disciplinas, ideologías y perspectivas para que ello ayude a avanzar y conseguir un consenso que nos haga ver los diferentes puntos de vistas que pueden darse respecto a dicho tema.
} 
mujeres, así como las graves vulneraciones de derechos a las que se encuentran sometidas en no pocas ocasiones.

Para finalizar, abogamos por una mirada interdisciplinar que incluya las voces de las protagonistas y los profesionales, pues ello podría contribuir a que los marcos normativos ampliaran los espacios de protección y seguridad para garantizar, en mayor medida, el derecho a la salud para la diversidad de mujeres que ejercen la prostitución. 
regiones en España", Revista de Estudios Regionales, 95, 2012, pp. 175-199.

\section{Referencias bibliográficas.}

ASAMBLEA GENERAL DE LAS NACIONES UNIDAS. Declaración Universal de los Derechos Humanos, París: ONU, $1948 . \quad$ Consultado en: http://www.ohchr.org/EN/UDHR/Documents/UDHR Trans lations/spn.pdf

BACIGALUPE, A. et. al. "Austeridad y privatización sanitaria en época de crisis: ¿existe diferencias entre las comunidades autónomas?" Gaceta Sanitaria, 30(1), 2016, pp. 47-51.

BURGAYA, J. El Estado de bienestar y sus detractores. A propósito de los orígenes y la encrucijada del modelo social europeo en tiempos de crisis. Barcelona, Octaedro, 2013, $256 \mathrm{pp}$.

CÁRITAS. La prostitución desde la experiencia y la mirada de Cáritas. Madrid, Cáritas Española Editores, 2016, 81 pp. Consultado en: http://www.caritasvitoria.org/datos/documentos/CARITASP rostitucion2016.pdf

COMISIÓN PARA REDUCIR LAS DESIGUALDADES SOCIALES EN SALUD EN ESPAÑA. "Propuesta de políticas e intervenciones para reducir las desigualdades sociales en salud en España”, Gaceta Sanitaria, 26(2), 2012, pp. 182-189.

CORTÉS, C. T. "Detesto que me digan puta", DonostiaSan Sebastián: Tercera Prensa = Hirugarren Prentsa, D.L., 2011, 287 pp.

ESTEBAN, M. L. "El Estudio de la Salud y el Género: Las Ventajas de un Enfoque Antropológico y Feminista", Salud Colectiva, 2(1), 2006, pp. 9-20.

FAURA MARTÍNEZ, U. \& LAFUENTE LECHUGA, M. "Evolución de los factores de riesgo de exclusión social por
GRAÇA, M. \& GONÇALVES, M. "Conhecimento, prática e ética: Os desafios da investigação-ação em contexto de prostituição feminina de rua", Revista Crítica de Ciencias Sociais, 108, 2015, pp. 135-156.

GOBIERNO DE ESPAÑA. Constitución Española, 1978. Consultado en:

https://www.boe.es/diario boe/txt.php?id=BOE-A-1978$\underline{31229}$

GOBIERNO DE ESPAÑA. Real Decreto-ley 16/2012, de 20 de abril, de medidas urgentes para garantizar la sostenibilidad del Sistema Nacional de Salud y mejorar la calidad y seguridad de las prestaciones, 2012. Consultado en: https://www.boe.es/buscar/act.php?id=BOE-A-2012$\underline{5403}$

JUNTA DE ANDALUCÍA. Atención a la salud de las personas que ejercen la prostitución, 2007. Consultado en: http://www.juntadeandalucia.es/salud/servicios/contenidos/a ndaluciaessalud/docs/31/AtencionProstitucion_FINAL.pdf

JUNTA DE ANDALUCÍA. Ley 16/2011, de 23 de diciembre, de Salud Pública de Andalucia, 2011. Consultado en: http://www.juntadeandalucia.es/boja/2011/255/4

JUNTA DE ANDALUCÍA. Carta de Derechos y Deberes en Salud, 2012. Consultado en: http://www.juntadeandalucia.es/servicioandaluzdesalud/con tenidos/derechos/DATA/SAS DyD ES.pdf

JUNTA DE ANDALUCÍA. IV Plan Andaluz de Salud, 2013. Consultado en: http://www.andaluciasana.es/PAS/links/IV PAS v9.pdf

JUNTA DE ANDALUCÍA. Anteproyecto de Ley de Garantías y Sostenibilidad del Sistema Sanitario Público de Andalucía, 2015. Consultado en: http://www.juntadeandalucia.es/export/drupaljda/cg/acuerdo 


\section{CEFD}

Cuadernos Electrónicos de Filosofía del Derecho

s/Anteproyecto $\% 20 \mathrm{de} \% 20 \mathrm{Ley} \% 20$ Sostenibilidad $\% 20$ Siste ma\%20Sanitario\%20junio\%202015.pdf

JUNTA DE ANDALUCÍA. "Actuación sanitaria con mujeres en situaciones especiales", Actuación Sanitaria ante la Violencia de Género, 2015, pp. 99-123. Consultado en:

http://www.juntadeandalucia.es/salud/export/sites/csalud/ga lerias/documentos/c_1_c 6 planes_estrategias/violencia_ge nero 2015/07 actuacion situaciones especiales.pdf

LÓPEZ PRECIOSO, M. \& MESTRE I MESTRE, R. M. Derecho de ciudadanía para trabajadoras y trabajadores del sexo. Valencia: Tirant lo Blanch, 2007, 278 pp.

MACKINNON, C. A. "Gender - The Future", Constellations, Vol. 17, № 4, 2010, pp. 504-511.

MAQUEDA ABREU, M. L. "La prostitución: el "pecado" de las mujeres"”, Cuadernos Electrónicos de Filosofía del Derecho, 35, 2017, pp. 64-89.

MARCOS DEL CANO, A. M. \& MÉNDEZ MARTÍN, J. "El derecho a la salud. Perspectivas tras la crisis", Revista de Derecho UNED, 19, 2016, pp. 151-182.

MINISTERIO DE SANIDAD, SERVICIOS SOCIALES E IGUALDAD. Plan nacional para la inclusión social del Reino de España 2013-2016. Madrid: Ministerio de Sanidad, Servicios Sociales e Igualdad, Centro de Publicaciones, 2014, 138 pp. Consultado en: https:/www.msssi.gob.es/ssi/familiasInfancia/inclusionSoci $\underline{\text { al/docs/PlanNacionalAccionInclusionSocial } 2013 \text { 2016.pdf }}$ MINISTERIO DE SANIDAD, SERVICIOS SOCIALES E IGUALDAD. Plan integral de lucha contra la trata de mujeres y niñas con fines de explotación sexual: 2015-2018. Madrid: Ministerio de Sanidad, Servicios Sociales e Igualdad, Centro de Publicaciones, 2015, 77 pp. Consultado en:

http://www.violenciagenero.msssi.gob.es/planActuacion/pla nContraExplotacionSexual/docs/Plan_Integral_Trata_18_Se ptiembre2015_2018.pdf

ORGANIZACIÓN MUNDIAL DE LA SALUD. "Constitución de la Organización Mundial de la Salud", Documentos básicos, $48^{a}$ Edición, OMS, 2014, pp. 1-21. Consultado en: http://apps.who.int/gb/bd/PDF/bd48/basicdocuments-48th-edition-sp.pdf $\#$ page $=7$

PANISELLO, M. L. \& PASTOR GOSALBEZ, M. I. "Salud con igualdad: una propuesta para la incorporación de la perspectiva de género en los sistemas sanitarios", Ciencias \& Saude Colectiva, Vol. 20(5), 2015, 1555-1565 pp.

PATEMAN, C. "What's Wrong with Prostitution?", Women's Studies Quarterly, Vol. 27, № 1/2, Teaching About Violence Against Women (Spring - Summer, 1999), pp. 5364.

PATEMAN, C. "Sex and Power", Ethics, Vol. 100, № 2 (Jan. 1990), pp. 398-407.

SEGURA BENEDICTO, A. "Recortes, austeridad y salud. Informe SESPAS 2014”, Gaceta Sanitaria, 28(S1), 2014, 7 $11 \mathrm{pp}$.

SILVA ARDANUY, F. M. Protección estatutaria de las políticas públicas de inclusión social en la CC.AA. de Andalucía. Valencia: Tirant lo Blanch, 2015, 91 pp. 